\title{
Mujeres argentinas y usos de drogas: análisis sociohistórico del uso femenino de sustancias psicoactivas en Argentina (1860-1930)
}

\author{
Argentinian women and the uses of drugs: A \\ sociohistorical analysis of female drug use in Argentina \\ (1860-1930)
}

${ }^{1}$ Autor de correspondencia. Doctora en Ciencias Sociales. Docenteinvestigadora, Instituto de Ciencias de la Salud, Universidad Nacional de Tres de Febrero, Ciudad Autónoma de Buenos Aires, Argentina. $\triangle$ (iD)

Victoria Sánchez Antelo

RESUMEN Este trabajo describe casos expuestos por expertos de los ámbitos legislativo y médico-legal periodístico, en los que se reporta el consumo de sustancias psicoactivas por parte de mujeres de Argentina, entre 1878 y 1930. Se presentan antecedentes sobre mujeres y usos de distintos fármacos, se analizan las intervenciones médicas que utilizan sustancias psicoactivas sobre el cuerpo femenino, y se detallan los casos de mujeres consumidoras desde las miradas expertas. En este periodo, los discursos expertos no buscaron comprender la especificidad femenina del consumo, sino promover el tema drogas como un problema. Esto se produce utilizando tres prototipos: la víctima de un marido enfermo, la prostituta que envicia a los débiles de espíritu (criminal nata), y la joven virtuosa que contraviene la ley del padre y sucumbe en la toxicomanía. Cada figura refuerza la necesidad de intervención estatal y control social.

PALABRAS CLAVES Abuso de Drogas; Prescripciones de Medicamentos; Legislación Médica; Identidad de Género; Argentina.

\begin{abstract}
This article describes cases presented by experts from the legislative and medical-legal fields regarding the use of psychoactive substances among Argentinian women from 1878 to 1930 . Background information is presented regarding the relationship between women and the use different drugs, medical interventions on the female body where psychoactive substances were used are analyzed, and experts' descriptions of cases of female drug users are detailed. Experts' discourses during this period did not attempt to comprehend the specificities of female consumption, but were rather used to position the issue of drug use as a social problem. This was done using three prototypes: the victim of a sick husband; the prostitute who encourages drug use among the weak in spirit (naturalborn criminals); and the virtuous young woman who succumbs to drug addiction in spite of her father's rule. Each figure reinforces the need for state intervention and increased social control.
\end{abstract}

KEY WORDS Substance-Related Disorders; Drug Prescriptions; Medical Legislation; Gender Identity; Argentina. 


\section{INTRODUCCIÓN}

En la Argentina de 1920, para introducir en el ámbito legislativo el tema drogas, se eligió exponer el caso de una sobredosis femenina. Los diputados de la Unión Cívica Radical (UCR) por la capital, José Roydero y Juan Capurro, lo utilizaron para exponer la relevancia de su proyecto de ley "Reglamentación del comercio de alcaloides" en la sesión del 31 de agosto de 1920 ${ }^{(1)}$. El caso elegido aportó múltiples dimensiones que fueron explotadas por los legisladores en sucesivas ocasiones. Se trató de una causa judicial emprendida contra un médico acusado de haber convertido a su mujer en morfinómana. Fue un caso resonado entre la burguesía porteña, ya que involucró a un médico discípulo de reconocidos galenos de la capital y a una mujer de la elite. Según el expediente judicial, el hombre comenzó a utilizar morfina luego de una fractura. Durante el viaje de recién casados en barco a Europa, la mujer se inició en el uso de morfina, la que fue facilitada y aplicada por el cónyuge ${ }^{(2)}$. En 1916, tras un uso recurrente de morfina, se produce la muerte de la mujer $^{(3)}$, motivo por el cual se inicia la causa judicial contra el marido médico ${ }^{(4)}$. Según Roydero, el documento aportado por la justicia: "es un expediente donde se ve a un hombre llevar a una mujer joven y hermosa al absoluto renunciamiento de su voluntad $y$, entregada a la suya, convertirla en un verdadero harapo humano"(5).

En Argentina, entre los médicos, el interés por los usos de los "alcaloides" se registraba ya a finales del siglo XIX. Sin embargo, este caso disparó el debate de expertos y de legisladores sobre el rol de los médicos en la administración de las sustancias, de los farmacéuticos en su provisión y, en particular, sobre el uso de sustancias psicoactivas por fuera de la supervisión médica y sobre la responsabilidad penal del toxicómano(6). En 1926, dicho debate culminó con la penalización de la tenencia sin prescripción de un conjunto de sustancias ${ }^{(7)}$.
A pesar del protagonismo femenino del caso utilizado por Roydero, en su exposición, así como la de otros, se presenta a la mujer solo como víctima pasiva sin detalles clínicos, al tiempo que se omite el proceso por el cual, en el arte de curar, se utilizaron esos mismos fármacos sobre el cuerpo femenino. Esta omisión posibilitó instalar figuras prototípicas para resaltar el peligro de una epidemia que, localmente no poseía una cuantificación clara. Las publicaciones académicas y legislativas del momento enfatizaron el peligro al que se exponía no solo a los ciudadanos, sino al propio futuro de la Nación. En dichas fuentes, si bien la mayoría de los casos narrados eran protagonizados por varones, se observa una mención asistemática de mujeres que utilizaron diferentes "alcaloides".

Los discursos moralizantes y clasistas, muy marcados al hacer referencia al consumo de sustancias por parte de mujeres, han sido centrales en los argumentos prohibicionistas que dieron forma a las políticas de drogas locales e internacionales ${ }^{(8,9,10)}$. Si bien las crónicas de comienzos del siglo XX hacen referencia a consumos femeninos, la interrogación sobre la figura de la mujer como consumidora de sustancias psicoactivas ha sido escasamente tratada. Incluso, en los estudios actuales se tiende a focalizar en el rol vinculado al tráfico más que a sus con$\operatorname{sumos}^{(11,12,13)}$, mientras los estudios de género resaltan la importancia de comprender los aspectos socioculturales asociados al uso de $\operatorname{drogas}^{(14,15,16,17,18,19)}$.

Por su parte, en la historiografía argentina no se ha realizado un análisis sistemático del consumo de sustancias psicoactivas por parte de mujeres. Algunos investigadores se han detenido en la conformación de los discursos psicopatológicos ${ }^{(6)}$, en los debates parlamentarios $^{(20,21)}$, así como en la figura de Leopoldo Bard y su articulación con los lineamientos políticos internacionales ${ }^{(7)}$. En este sentido, la investigación que da lugar a este artículo utilizó fuentes ya analizadas por estudios previos, pero colocando el foco en los casos de mujeres consumidoras. 


\section{ASPECTOS METODOLÓGICOS}

El objetivo de la investigación fue, por tanto, describir los casos expuestos por expertos de los ámbitos legislativo y médico-legal de mujeres que utilizaron sustancias psicoactivas en la Argentina entre 1860 y 1930 . Se analizaron discursos expertos del período, mostrando que los mismos no buscaron definir la especificidad femenina, sino instalar el "tema de las drogas" como un campo problemático que requería intervención estatal y mayor control social. Dar cuenta de ese proceso histórico implica un aporte a la comprensión de la política actual en materia de salud mental, legislación penal y del accionar estatal sobre las personas que usan drogas, particularmente, sobre las mujeres.

Cabe aclarar, en primer lugar, que se utiliza el concepto de sustancias psicoactivas, o "drogas" como su sinónimo, para hacer referencia al conjunto de sustancias que poseen efetos psicoactivos sobre el sistema nervioso central en el cuerpo humano, independientemente de su estatus legal. Esto incluye el alcohol, pero también productos como cocaína, morfina, láudano, cáñamo indiano, bicloruro de mercurio, cloroformo, éter o nitrito de amilo.

En segundo lugar, la delimitación temporal del análisis buscó recorrer el período histórico en el cual alguna de esas sustancias pasaron de utilizase sobre el cuerpo femenino en la medicina local y venderse sin necesidad de prescripción, a transformarse paulatinamente en un problema de salud pública que requería su prohibición y la intervención estatal a través del accionar médico-policial(20).

En tercer lugar, se utilizaron fuentes documentales publicadas entre 1860 y 1930, en las que la temática refería al consumo de sustancias psicoactivas en seres humanos y contenían referencias sobre uso de drogas por parte de mujeres argentinas. Cabe aclarar que, en las publicaciones académicas, los autores locales, además de un desarroIlo teórico sobre el tema que trataban, en la configuración del cuadro clínico reproducían casos reportados por académicos europeos, así como por autores estadounidenses para justificar modelos de reglamentación estatal.

En las tesis analizadas de los médicos argentinos se reproducen casos descriptos por los teóricos europeos como Benjamín Ball, de Jean-Baptiste Fonssagrives, Eduard Levisntein, Jean-Martin Charcot o Henri Legrand du Saulle, entre otros. Esa reproducción sirve a los argentinos para presentar los elementos teóricos de su análisis. De sus tesis, para esta investigación solo se consideraron los casos observados de mujeres en Argentina. Asimismo, en la recopilación periodística realizada por Leopoldo Bard, se reproducen artículos de la prensa europea en los que se describen casos policiales en los que la toxicomanía es el hilo conductor. También cita al propio Harry Anslinger, quien describe la situación en EEUU. En este sentido, los casos reportados por Bard, mencionados en la prensa francesa o estadounidense, se excluyeron del análisis. Si bien esas referencias se tomaron en cuenta para comprender la perspectiva experta sobre el tema, para el análisis se recuperaron los casos referidos a mujeres argentinas que consumieron algunas de estas sustancias.

En cuarto lugar, los acervos consultados para esta investigación fueron: la biblioteca de la Facultad de Medicina de la Universidad de Buenos Aires (UBA), donde se accedió a revistas científicas y tesis doctorales publicadas entre 1860 y 1930; los diarios de sesiones legislativas entre 1894 y 1933 de la Honorable Cámara de Diputados de la Nación y del Honorable Senado de la Nación, y publicaciones de divulgación médica del mismo período, archivados en la Hemeroteca de la Biblioteca del Congreso de la Nación; y los números publicados entre 1898 y 1930 de la colección digital Caras y Caretas, edición de Buenos Aires, de la Biblioteca Nacional de España.

Este trabajo es parte de un programa de investigación en género y usos de drogas, orientado a analizar el proceso histórico de producción de saberes y problematización del consumo femenino de drogas en el campo de la salud y la política argentina. 
El presente artículo se divide en tres partes. La primera, describe antecedentes sobre la relación entre mujeres y usos de distintos fármacos. Luego se analizan las distintas intervenciones médicas sobre el cuerpo femenino mediante la utilización de sustancias psicoactivas y las consecuencias de ese uso. Finalmente, se exponen los casos de mujeres consumidoras, descriptos por las miradas expertas, para la justificación de procedimientos médicos, reformas legales e institucionalización de medidas políticas. Cada parte, corresponde a momentos de transformación de los discursos analizados. En todas las citas textuales se respeta la sintaxis y gramática original de las fuentes consultadas.

\section{MUJERES EN EL ARTE DE CURAR DEL VIRREINATO: LAS GENTES VULGARES Y CUATRO YERBAS}

El vínculo entre las mujeres y las drogas no se limita al rol de estas como consumidoras. El uso de hierbas, tónicos o elixires, así como el ejercicio de prácticas curativas, las han tenido como protagonistas a lo largo de la historia ${ }^{(22)}$. La institución del rol médico y farmacéutico exigió una fuerte regulación de prácticas domésticas y, específicamente, la limitación para producir y administrar remedios caseros. El proceso de modernización del arte de curar ha requerido un desplazamiento del conocimiento y poder femenino en la administración de las distintas sustancias mediante el descrédito ${ }^{(23)}$. Dicho proceso se produjo de forma compleja, desigual, marcado por avances y retrocesos, según el mayor o menor éxito de los fármacos legalizados para curar las enfermedades ${ }^{(24)}$. En el Río de la Plata, ya en la época virreinal, se registraba como problema público la relación entre mujeres y los usos de fármacos. Según documentó Juan Ramón Beltrán ${ }^{(25)}$, mujeres indígenas utilizaban diversas hierbas nativas y preparados con fines curativos. Estas prácticas las definió como "curanderismo indígena propio de pueblos primitivos" y las ubicó como parte de un confuso grupo de actividades orientadas al engaño, basadas en el exorcismo y la hechicería. Hacia 1790, esas actividades estaban tan extendidas que los representantes de la corona española alertaban que las

...gentes vulgares del País, opuestas a Médicos y Medicinas de Botica, entregándose con más facilidad a aquella clase Empíricos, que reducen su ciencia a cuatro yerbas... y algunas recetas mal compuestas, y peor indicadas, que Ilaman remedios caseros...

El desamparo, y la escasez que ay de ellos en los demás Lugares da motivo á que aficionados a esta facultad, o Vnas mujeres que la piedad o interés les compele apliquen remedios sin conocimiento de los síntomas de los accidentes que toleran. (Citado por Beltrán en Historia del Protomedicato de Buenos Aires, 1937) ${ }^{(25)}$

Los rótulos utilizados por Beltrán sintetizaban la disputa entre la medicina tradicional o nativa y el avance colonizador de lo que Di Liscia denominó las "corrientes tecno-culturales europeas". Esto supondrá, por un lado, el desplazamiento del rol de las mujeres en la medicación de comunidades y familias y, por otro, un doble proceso: la apropiación de los conocimientos indígenas en el manejo de hierbas y pociones, así como el relego de sus saberes a un lugar de inferioridad. Esa jerarquización posibilitó consolidar las técnicas y las concepciones traídas del continente europeo como verdades incuestionables ${ }^{(26)}$.

Asimismo, la modernización supuso imponer sistemas clasificatorios, transformación registrada en el Código Penal redactado por Carlos Tejedor en 1867. Allí se introdujeron regulaciones sobre la elaboración de bebidas y comestibles, asociadas a la necesidad de penar la adulteración de sustancias que devengan nocivas para la salud ${ }^{(27)}$. Se trató no solo de la clasificación y el ordenamiento de sustancias en alimenticias, curativas y nocivas, sino también de la diferenciación de profesiones, actividades lucrativas y usos legales 
e ilegales. La modernización del arte de curar implicó excluir de la institución de prescribir y preparar fármacos a prácticas terapéuticas y actores. Englobadas bajo el rótulo de curanderismo y charlatanería, muchas de estas actividades eran protagonizadas por mujeres y los legisladores comenzaron a demandar su control $^{(26,28,29,30,31)}$. Hacia finales del siglo XIX, las mujeres reducidas al rol materno fueron reubicadas como promotoras de la salubridad en el hogar, como cuidadoras auxiliares de las indicaciones expertas, implementadoras de las medidas preventivas y supervisoras de enfermos ${ }^{(24)}$.

\section{EL CUERPO FEMENINO: TERRITORIO DE ACCIÓN Y PRESCRIPCIÓN DE SUSTANCIAS}

Entre 1870 y 1890 , la transformación de la técnica médica en Europa se puede atribuir, en gran medida, a la urgencia por dar respuesta a elevadas tasas de mortalidad durante el parto y el puerperio. La generalización de estos avances técnicos trajo aparejada la extensión del uso de medidas de asepsia, así como la utilización de la sutura y de anestésicos como la morfina ${ }^{(32)}$. Los expertos argentinos no estaban ajenos a dichos avances, los cuales fueron citados y reproducidos en sus publicaciones.

Emulando a sus pares europeos, los argentinos impulsaban la medicalización del embarazo. Una tesis publicada en 1908, se focalizaba en la higiene durante el período de gestación e incluía recomendaciones sobre los hábitos alimenticios, el control de la embarazada contra el impulso de usar bebidas alcohólicas, indicaciones sobre la vestimenta y medidas de asepsia en la vagina con bicloruro de mercurio(33).

Desde finales del siglo XIX e inicios del siglo XX, se extendió el uso de la cocaína, el extracto de opio o la inyección de morfina para atender síntomas en los cuerpos femeninos. En 1895, estos fármacos se indicaban para el manejo de los vómitos incoercibles ${ }^{(34)}$, cuadro que debilitaba a la embarazada, con efectos negativos sobre el feto. Se prescribían también para los dolores del parto y como anestesia en la cesárea vaginal, que según una tesis de 1918, era una práctica utilizada en el país desde $1907^{(35)}$.

El médico Luis Villarroel, en 1903, reclamaba en su tesis trastocar "la inviolabilidad de la preñez [...] impuesta por la religión" y promover el control médico del embarazo y del parto para luchar contra "las falencias de la naturaleza". Si bien sugería evitar matrimonios entre personas con "enfermedades o taras hereditarias", frente a un embarazo ya en curso la intervención médica se presentaba "como la opción lógica, racional y hasta cierto punto científica" (36).

En este contexto, el auge de los fármacos se extiende y en 1915, el reconocido médico tucumano Eliseo Cantón incursionaba en el perfeccionamiento de anestésicos, creando la partoanalgia, alabada por los miembros de la Academia Nacional de Medicina. En una nota en la revista de divulgación Caras y Caretas del mismo año, Cantón exponía: "cuatro centigramos [de clorhidrato de morfina] bastan para suprimir los dolores del parto y seis para determinar la analgesia quirúrgica en la mayoría de los casos de ginecología"(37).

En el siglo XIX, las corrientes hegemónicas de pensamiento médico parecían detenerse con énfasis en la fisiología y en la moral femenina ${ }^{(38)}$. En América Latina, hacia comienzos del siglo XX florecieron las ideas eugenésicas, cuya atención estuvo en las políticas de control de la reproducción y en las conductas sociales de varones y mujeres que afectaran ese control. Sus planteos promovieron la injerencia del Estado, asistido por una creciente medicalización y la aplicación de regulaciones legales, sobre asuntos privados $^{(30,39,40,41)}$.

Durante el período analizado se observaron profundos cambios sociodemográficos, políticos y económicos en Argentina y, en especial, en la Ciudad de Buenos Aires. La creciente inmigración de ultramar y la crisis económico-política exacerbaron la conflictividad social. Desde los saberes expertos, las ideas eugenésicas se dieron como reacción a las tensiones derivadas de la desigualdad. El 
determinismo biológico impregnó gran parte de las conceptualizaciones sobre la salud y la enfermedad, sobre el rol del campo médico como actor político y del Estado en el control de distintas prácticas individuales ${ }^{(42,43)}$. Se configuró así una biopolítica recubierta por el discurso cientificista, pero cuyo anhelo no fue otro que definir acciones políticas orientadas a institucionalizar jerarquizaciones de clase, de geografía y de género sobre argumentos biológicos ${ }^{(42)}$. En términos de género, los discursos expertos contribuyeron a legitimar las jerarquías del modelo patriarcal ${ }^{(44,45)}$.

En Argentina, esta corriente se entrelazó con los debates en torno al crecimiento demográfico como factor de crecimiento económico y la inquietud de las elites con relación a la "calidad" de la población. Para los médicos, comenzaban a diferenciarse un conjunto de padecimientos específicos del cuerpo femenino que gravitaban sobre la especificidad de su sexo y su sexualidad. Entre las "enfermedades de mujeres", la clorosis y el vaginismo se presentaban como patologías a las cuales se debía prestar especial atención. Asociadas a manifestaciones del proceso de transformación del cuerpo femenino, para los médicos argentinos resultaban alarmantes y eran abordadas como indicios de una pasión que, mal administrada, debía concebirse como patológica ${ }^{(46)}$.

En el caso de la clorosis, en 1847, el médico argentino Adolfo Peralta ya reportaba en su tesis los experimentos europeos que conectaban la deficiencia de hierro con dicho cuadro clínico; al tiempo que, entre los factores etiológicos, mencionaba afecciones morales, fruto de amores contrariados ${ }^{(47)}$. Aun así, el autor criticaba a los galenos que definían la clorosis como una especie de histeria y rechazaba el uso que hacían de los narcóticos para su tratamiento ${ }^{(47)}$. Respecto al vaginismo, la obra de divulgación titulada Mi médico: Guía práctica de Medicina e higiene, lo definía como

...una excitabilidad, una sensibilidad excesiva de los órganos genitales... Se observa en ciertas mujeres jóvenes, nerviosas, excitables, en ocasiones hasta histéricas... sobre todo, en las recientemente desposadas. Su tratamiento deberá estar orientado a "luchar contra el elemento nervioso". (Fournol, Heiser y Samne, 1930) $^{(48)}$

El interés sobre la calidad demográfica impulsó las iniciativas para la prevención de los "venenos sociales", en particular, la sífilis, la tuberculosis y el alcoholismo, considerados factores con efecto sobre la herencia $y$, por tanto, materia de intervención médica ${ }^{(42,43)}$. La atención de la higiene y la terapéutica genital femenina para su tratamiento, requirió el desarrollo de técnicas, como la disposición del cuerpo para el reconocimiento médico, así como de tecnologías específicas, como las inyecciones de líquidos o gases. En el tratamiento de la sífilis, en el año 1882, Miguel Figeroa mencionaba la bicloruro de mercurio en la vagina de las embarazadas, aún a riesgo de provocar abortos o "inducir el saturnismo", fruto de la intoxicación con mercurio $^{(49)}$; mientras que, en 1887 , otro médico reportaba el uso de preparados de opio, supositorios de cocaína o belladona para luchar contra el "elemento nervioso" que originaba el vaginismo ${ }^{(50)}$.

La sintomatología de las "enfermedades de mujeres" pivotó entre la naturalización de la debilidad y la delicadeza, como características propias de lo femenino, y la patologización de su condición, al tratarla como fruto del exceso de energía pasional que debilitaba su espíritu moral ${ }^{(22,51,52,53)}$.

La hipótesis orgánica de la debilidad y la delicadeza femenina no solo fue patrimonio de los médicos. Los juristas argentinos también la utilizaron para explicar la inferioridad femenina frente a la ley penal. En aspectos orgánicos encontraron la dificultad para el control de las pasiones, que explicaban una menor adhesión al orden moral. Así en la tesis publicada en 1878 sobre Moralidad Comparada del hombre y la Muger bajo el punto de vista penal, el doctor José Calderón sostenía:

Las pasiones estremadas son todavía más delirantes en la muger que en el hombre; porque este vive mas bajo la influencia 
de su cerebro y por consiguiente de su voluntad, y la muger bajo la influencia del sistema nervioso ganglionar, es decir, bajo el predominio del sentimiento, que no raciocinia. (Calderón, 1878) ${ }^{(54)}$

La articulación entre debilidad físico-moral y el peligro de la degeneración fue la trama explicativa desde mediados del siglo XIX y parte de las primeras décadas del siglo $X X^{(22)}$. Cabe detenerse en el concepto de degeneración ya que fue la utilizada por los expertos argentinos en el análisis de las toxicomanías. Según Benedict Morel, la naturaleza humana primitiva, en el sentido de no corrompida, se caracterizaba por la adhesión "natural" a la ley moral. Distintas enfermedades mentales, concebidas como afecciones morales, se explicarían por la constitución anormal del organismo, condición hereditaria acumulativa, que conducía a un proceso de desviación morbosa de la raza. Valentín Magnan, por su parte, reafirmó el factor biológico como variable explicativa al establecer que los daños en áreas del cerebro daban lugar a la falta de voluntad moral(4). Dichos daños orgánicos producían el desequilibro psíquico y se debían a secuelas generadas por enfermedades, emociones, trastornos fisiológicos o debilitamiento físico ${ }^{(55)}$.

En esta línea, el destacado e influyente médico psiquiatra argentino Domingo Cabred analizó cuadros clínicos a los que denominó "locura refleja". Según su tesis de 1881, la alienación mental era un reflejo de la falla orgánica. En el caso de las mujeres, este cuadro se derivaba de los cambios en su aparato genital, en las fases del ciclo ovular y, particularmente, durante el puerperio. Entre 1876 y 1880 , alrededor del $3 \%$ de los casos ingresados en La Convalecencia, antiguo hospicio de mujeres de la Ciudad de Buenos Aires, respondían a "locura originada por el puer-

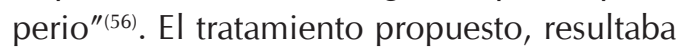
de la combinación de intervenciones sobre el órgano que originaba la patología e inyecciones de morfina para atender la sintomatología como delirios, insomnio o alucinaciones.

Cabred, en sus tesis, describe el caso de Eufemia para explicar su razonamiento.
La mujer, ingresada en La Convalecencia en 1879 , era argentina, soltera, de 45 años, sin antecedentes familiares de alienación ni de enfermedades que explicasen la crisis psíquica. Luego de sufrir un violento golpe, presentó una hemorragia uterina, alucinaciones de la vista y el oído, así como delirios persecutorios. Aun sin contar con antecedentes familiares de degeneración, la mujer sufría de períodos de "violenta agitación" durante la fase menstrual. El repetido insomnio agravaba su cuadro, para lo cual Cabred prescribió uso de extracto de cannabis índica y shock térmico a través de baños fríos, una práctica muy extendida en la época ${ }^{(56)}$.

Entre los médicos argentinos, el detaIlado análisis de rasgos hereditarios fue central en la configuración de la anamnesis para la definición psicopatológica, en general, y de la toxicomanía, en particular ${ }^{(26,57)}$.

\section{La histeria como campo de acción farmacológico}

Un conjunto de síntomas, como el nerviosismo, las pasiones no controladas, la debilidad física y moral, se entrelazaban con la figura de la histeria que, sin ser exclusivamente femenina, se institucionalizaría como inherente a su subjetividad ${ }^{(58)}$. Leída como un cuadro a medio camino entre lo psicopatológico y la simulación, su estudio devino en una "espectacularización del dolor" de la mujer moderna ${ }^{(22,51,53,59)}$.

Hacia finales de la década de 1880, aparece con mayor claridad el interés de la medicina argentina por la histeria. Siguiendo a Vallejo ${ }^{(58)}$, se pueden identificar dos posturas en las tesis de los médicos argentinos: una, que se focalizaba en la emotividad, el nerviosismo y la moralidad para la identificación del cuadro; y, otra, que se esforzó por identificar rasgos fisiológicos para explicar el origen a la patología. Ambas lecturas sobre la histeria coincidieron en el uso de alcaloides para atender la sintomatología.

Quienes ponían el foco en el factor emotivo y moral, proponían el casamiento de la histérica como tratamiento. Según Ignacio 
Firmat, en su tesis de 1889, esta indicación apuntaba a fortalecer el carácter, disciplinando el nerviosismo bajo el mando del marido, y así contrarrestar la debilidad moral; al tiempo que permitía calmar las "inquietudes enfermizas" y la "afectividad inquieta", propia de muchas mujeres jóvenes. Aun así, sugería no dar lugar "al desahogo muy vívido" de la pasión en las mujeres, ya que eso daría lugar a "conmociones violentas y excitaciones inmoderadas"

Siguiendo a autores franceses, como Jean Martin Charcot y Henri Legrand du Saulle, los argentinos proponían también tratamientos como la ducha de agua fría y, según Firmat, "fustigar a la paciente con una toalla mojada"(60), aplicación de electricidad y exhalaciones de cloroformo o nitrito de amilo. Esto llevaba a la necesidad de contrarrestar los "efectos esitantes de algunos fármacos", como del nitrito de amilo, para lo que se prescribía éter, opio y sus derivados (morfina y láudano), aun cuando otros médicos advertían sobre el peligro de "que se apasionen por estos anestésicos"(60). Según, el médico Celestino Arce, en 1881, en el Manicomio de Mujeres de Buenos Aires se evidenciaba el uso "en gran escala" de píldoras de extracto de opio para procurar el sueño e inyecciones de morfina, ya que producían resultados satisfactorios para "moderar la excitabilidad del sistema nervioso"(61).

Entre quienes dieron mayor preponderancia al elemento orgánico de la histeria, se puede mencionar al médico Arturo Ferrand y a Juan Yzaurralde. El primero, en su tesis de 1888, establecía que el origen de la patología se debía a "la falta de armonía o desequilibrio entre el elemento nervioso voluntario o cerebral y el elemento nervioso involuntario o espinal". La prevalencia de la patología entre las mujeres se debía a que estas poseían "una organización más débil y un sistema nervioso más desarrollado, es más apta para ser víctima de este mal"(62). Asimismo, establecía que el médico debía combatir la sintomatología corporal como las diarreas, las convulsiones y el ataque histérico, utilizando éter, cloroformo e inyecciones de morfina ${ }^{(62)}$.
En el mismo año que Ferrand, Juan Yzaurralde, para el desarrollo teórico de sus tesis, puso el énfasis en las explicaciones que ubicaban el origen de la histeria en el cerebro y en los ovarios. Aun así, en las observaciones realizadas en el Manicomio de Mujeres de Buenos Aires, la etiología señalaba confusamente aspectos orgánicos y emotivos. Entre las observaciones realizadas por Yzaurralde, llaman la atención casos de niñas y jóvenes, las cuales presentaban crisis violentas y ataques convulsivos histéricos. En Josefa, de 14 años, el médico atribuía estos síntomas al retraso en la primera menstruación y antecedentes de familiares de alcoholismo; en María, de 17 años, la crisis había sido originada por un amor contrariado; mientras que en L.B., de 16 años, resultaron determinantes los antecedentes familiares (una hermana con ataque de nervios). En el caso de Juana, argentina de 30 años, de "temperamento bilio-nervioso", Yzaurralde no logró identificar antecedentes hereditarios u orgánicos, solo un disgusto familiar que desencadenó el cuadro histérico. A pesar del origen dispar de los síntomas, a todas se le prescribió baños fríos, inyecciones de morfina y "medicación reconstituyente" ${ }^{\prime(63)}$.

De forma independiente de la etiología de la histeria, estos fármacos fueron considerados los verdaderos "antihistéricos". Aun así, Firmat alertaba que la decisión sobre la cantidad y la frecuencia de las aplicaciones, así como el instrumental para las inyecciones, no debían dejarse al alcance de la paciente "ya que el alivio que les produce las arrastra a la multiplicación de las dosis". En este sentido, afirmaba que "la intoxicación supervisada" era la única que previene la tolerancia y la morfinomanía ${ }^{(60)}$.

\section{Intoxicaciones y morfinomanía}

En las decripciones médicas, la histeria, los agentes intoxicantes y la morfinomanía, quedaban entrelazados de forma confusa. En 1900, Eduardo Doyle articulaba en su tesis la intoxicación por bicloruro de mercurio y la histeria. Como se ha mencionado, este preparado se utilizaba en el tratamiento de la sífilis, 
patología más prevalente entre las mujeres sometidas a la prostitución. Según señalaba Doyle, el bicloruro de mercurio junto con el uso de alcohol funcionaban como catalizadores de un cuadro específico: la histeria tóxica. Si bien la causa primera era la predisposición hereditaria, para este médico, una intoxicación podía despertar la histeria latente ${ }^{(64)}$.

Por su parte, Antonio Almeida, en 1891, presentaba su tesis sobre la Morfinomanía como cuadro clínico específico. La etiología del cuadro, llamativamente, la ubicaba en estilos de vida y en sectores sociales específicos:

...las exigencias de la vida moderna que mantienen á las personas en perpétua agitación nerviosa, [...] el neurosismo tan frecuente y generalizado en las altas clases social, la imitación y el contagio, el escepticismo de nuestra generación unido á esa sed insaciable de sensualismo que trae pronto el agotamiento $y$ el hastío. (Almeida, 1891) ${ }^{(65)}$

Almeida reconocía el entusiasmo médico por el uso de las sales de morfina, ya que resultaba efectivo contra dolores e incluso para curar "enfermedades morales". Sin embargo, alertaba sobre las consecuencias de dejar en manos de los enfermos o de sus parientes la administración de la droga. Según su opinión, la inexperiencia de sus colegas con la morfinomanía solía llevarlos a confiar en el paciente, dejando a su disposición el fármaco y "abriendo así al vicio". También introducía una crítica al gremio farmacéutico, la cual sería repetida por los legisladores años después: la venta sin restricción y sin control riguroso de la autenticidad de la prescripción médica era un facilitador de la intoxicación ${ }^{(65)}$.

De las seis observaciones realizadas en Buenos Aires, Almeida registró en 1890 solo una mujer. El caso de una "Señora distinguida" que presentaba un "acceso de histeria" y fue ingresada "al Instituto Frenopático". La anamnesis indicaba que la mujer de 37 años no presentaba antecedentes hereditarios que justifiquen el cuadro, aunque fue caracterizada como "de buen carácter, pero impresionable y nerviosa". El rasgo más Ilamativo fue que, para mitigar dolores de su último embarazo, la mujer había utilizado inyecciones de morfina, extendiendo su aplicación e incrementando las dosis, incluso luego del parto ${ }^{(65)}$. El origen de la morfinomanía no se explicaba como iatrogenia, sino por la disponibilidad del fármaco sin supervisión médica.

Este caso, como otros, evidenciaba los efectos producidos por el uso de fármacos considerados esenciales para la práctica médica ${ }^{(4)}$. Hacia 1923, Leopoldo Bard, médico, diputado de la capital por la UCR y uno de los promotores de la reforma legislativa, publicó en la Revista de la Asociación Médica Argentina, resultados de una encuesta realizada a personalidades de la medicina local y del exterior. Allí, corroboró que figuras destacadas de las ciencias médicas en Argentina, como José Arce, Carlos Udaondo y Mariano Castex, alertaban sobre lo difícil que sería prescindir de la morfina y la cocaína para los tratamientos. Incluso, uno de ellos, contradecía uno de los argumentos centrales de Bard, al afirmar que la toxicomanía en Argentina se observaba en muy pocos $\operatorname{casos}^{(66,67)}$.

En este sentido, la tesis de Almeida aportaba, además, un aspecto clave. En su análisis, utilizaba el alcoholismo como modelo de referencia para conformar el cuadro psicopatológico de la morfinomanía. Ese paralelismo fue nodal en los argumentos de legisladores ya que, en el contexto de una "epidemia alcoholista", la prohibición de los alcaloides se presentaba como la acción preventiva más lógica antes que se produzca su difusión.

\section{LAS MUJERES QUE USAN ALCALOIDES: VÍCTIMAS O CULPABLES}

Las primeras décadas del siglo XX estuvieron marcadas por transformaciones en las relaciones de género, fenómeno evidenciado en los cambios demográficos como la disminución de la tasa de natalidad. En un contexto de ampliación de los sectores medios urbanos, 
en la ciudad de Buenos Aires, las mujeres ocuparon espacios en la vida pública antes impensados ${ }^{(68)}$. Estos procesos convocaron el análisis de distintos expertos, quienes hicieron de las conductas femeninas el objeto de sus desarrollos. La matriz institucional establecida en la época, privilegió el avance del control estatal a través de médicos y policías, preservando la moral colectiva por sobre las libertades y la autonomía individual, en especial cuando se trató de mujeres ${ }^{(69)}$.

En ese marco, en 1920, se presentó en la Cámara de Diputados de la Nación Argentina, el proyecto ya citado, orientado a la "Reglamentación del comercio de alcaloides". Tal y como sintetizó el médico Gregorio Berman en $1926^{(70)}$, en ese debate quedaban delimitados dos modelos en tensión: uno, con foco en la venta de estos productos y, otro, con énfasis en el consumo. Este último modelo ancló sus argumentos en la "debilidad de espíritu" del consumidor y en el papel de los galenos, facilitando el acceso a los fármacos. En las exposiciones de los legisladores, muchos de ellos también médicos, se utilizaba una delimitación inespecífica del fenómeno: era tanto un "vicio elegante" como una "costumbre exótica". La etiología la ubicaban en la moral de las conductas, en "la situación cómoda de no hacer nada: la pereza que engendra todas esas situaciones anormales". La supervisión de quienes consumían los alcaloides se demandaba como necesaria, ya que con el libre uso no solo se degeneraban a sí mismos, sino que hacían peligrar el futuro de la sociedad en su conjunto, degenerando la raza (exposición de motivos del diputado Roydero, el 31 de agosto de 1920) $)^{(5)}$.

Era el comienzo de un proceso de cambio a partir del cual los usos de algunos fármacos serían conceptualizados como "ilegítimos" $"(7,20)$. Esto daba origen a mutaciones discursivas sobre el rol de los médicos y los farmacéuticos -facilitando el acceso a las sustancias psicoactivas ${ }^{(20)}$ - y también sobre la definición de "víctimas y victimarios". Ya en 1919, un decreto del Departamento Nacional de Higiene restringía la venta sin prescripción facultativa de cocaína, morfina, éter, cáñamo indiano y sus derivados ${ }^{(3)}$. Esa medida puso en evidencia otros procesos que alarmaron a los legisladores. El diputado Capurro, en su intervención del 10 de junio de 1920, resaltaba con espanto que
La aplicación del decreto [del Departa- mento Nacional de Higiene] comprobó dolorosamente que algunas consumido- ras eran niñas menores de edad. No faltó tampoco la nota de una pobre mujer que Ilorando se presentó a las puertas del despacho a solicitar permiso para que se le vendiera cocaína, que por la prohibi- ción decretada no conseguía libremente. (Exposición de motivos del 10 de junio de 1920) ${ }^{(5)}$

La referencia a figuras como niñas y mujeres reflejaba los esfuerzos de los legisladores para resaltar el daño que producía la legalidad de algunas prácticas, aun cuando las estadísticas no reflejaban semejante alarmismo. Sin embargo, el proceso no fue lineal, ya que la introducción de una prohibición como la pretendida involucraba numerosas dimensiones a contemplar. Mientras los legisladores de la UCR defendían la prohibición para evitar los usos ilegítimos de fármacos, los representantes del Partido Conservador por la provincia de Buenos Aires, contraargumentaban a su favor por tratarse de productos clave en la industria, como era el caso del éter (intervención del diputado Silvio Parodi, del 1 de septiembre de 1920)(5). El listado de fármacos y productos a incluir en la prohibición estaba en plena definición y los criterios para definirlos como "problemáticos" también ${ }^{(20)}$. El diputado Marcial Quiroga, representante de San Juan por la UCR, proponía en la sesión del 1 de septiembre de 1920, incluir al hachich, "este soñador eterno", y en su argumento enfatizaba "que en las mujeres produce trastornos"(5). También denunciaba los efectos de las pastillas de bicloruro de mercurio, utilizadas en el tratamiento de la sífilis, tanto para varones, mujeres y niños, que durante el embarazo provocaba clorosis aguda y hemorragias. Según el diputado, entre 1915 y 1920, en el "hospital Ramos Mexia" (sic) de la capital se habían registraron 240 mujeres 
intoxicadas con pastillas de bicloruro de mercurio, muchas de ellas jóvenes de 15 a 22 años, algunas "parturientas, siendo así causa de infanticidios" (intervención del diputado Quiroga, en el debate del 1 de septiembre de 1920$)^{(5)}$. La definición sobre la legitimidad de los usos se tornaba gris: mientras, por un lado, era extendida la prescripción de esos productos durante el embarazo para prevenir la degeneración originada por la sífilis, por el otro, se observaba que algunas mujeres hacían una aplicación abusiva de drogas con fines ajenos a la terapéutica.

A comienzos de la década de 1920, la definición del papel de la mujer, reducida a la condición de madre, logró hacer coincidir a laicos y católicos: para unos, garantizaba la fuerza de trabajo necesaria para el futuro de la patria, para otros, la reproducción de los valores morales católicos ${ }^{(53)}$. Fue un periodo en el que el análisis de la figura de la mujer, como objeto de los discursos expertos, estaba guiado por las teorías eugenésicas, para las que las funciones maternales y reproductoras eran centrales ${ }^{(38,69)}$.

Aun cuando los procesos políticos tendientes a debatir, redefinir y cuestionar el rol de la mujer en la sociedad argentina tenían ya algunas décadas, para la mayoría de las mujeres, el ideal de normalidad femenina estaba circunscripto a las tareas del ámbito privado y fuertemente vinculado al rol maternal. La creciente presencia en el mercado de trabajo, así como la demanda de acceso a la educación y a derechos civiles, tensionaba este ideal ${ }^{(71)}$. Desde la mirada médica, dominada por varones, los modos de conducirse como los hábitos y la vestimenta, resultarían un foco de atención y control moral. La visibilidad de la mujer en el espacio público y, en especial algunas prácticas, fueron un indicador de su peligrosidad y degeneración ${ }^{(53,68,72)}$.

Los casos introducidos por Leopoldo Bard, en su promoción del prohibicionismo, fueron delimitando dos perfiles de la mujer que utilizaba drogas, diferenciados por la clase social: un fue la mujer de las familias distinguidas que, víctima de un marido degenerado, devenía toxicómana; otro perfil, fue la mujer de los sectores subalternos, la prostituta, definida como una "criminal nata" que arrastra a los varones de la elite, "débiles de espíritu", al consumo.

\section{El caso de un degenerado superior y la degradación de un espíritu cultivado}

El caso había sido descripto en 1918 en un trabajo publicado en la Revista de Criminología, Psiquiatría y Medicina Legal, bajo el título "La morfinomanía ante la ley penal”(2,6), citado por el diputado Roydero en la sesión del 31 de agosto de 1920 y retomado por Bard en su obra Los peligros de la Toxicomanía, publicada en 1923. Luego de introducir su propuesta de reforma del código penal, Bard reproduce el auto judicial del proceso, en el que se describía a la víctima como

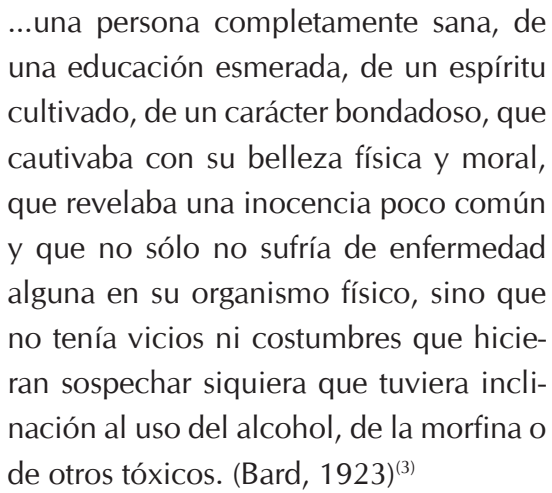

Según constaba en el expediente, se trataba de un matrimonio de los sectores medios acomodados, en el que el factor explicativo de la morfinomanía en la mujer era el diagnóstico del marido como "degenerado superior". De acuerdo a la clasificación de Magnan ${ }^{(57)}$, ese cuadro correspondía a uno de los cuatro tipos de degenerados. Entre los rasgos destacados de este subtipo estaba la "apariencia normal e inteligente", que por un desequilibrio neurofisiológico había desencadenado el déficit de voluntad. El marido "no solo era médico, sino que llegó a jefe del servicio", con lo que su inteligencia era innegable, pero por herencia degenerativa y eventos que agravaron su cuadro, dio curso a conductas degeneradas como la imposibilidad para controlar el consumo de morfina ${ }^{(57)}$. 
La relevancia del caso está en la articulación de la figura del médico descarrillado de su deber como "salvador de la humani$\operatorname{dad}^{\prime \prime(71)}$, con la perturbadora figura del "médico indigno" que hace un uso deshonesto de su poder ${ }^{(7)}$. Coherente con esto, parte de las reformas promovidas por Bard, apuntaron a la reglamentación del comercio y el mayor control de las profesiones médicas y farmacéuticas.

A pesar de concluirse el caso con la muerte de la mujer, Ilama la atención cómo los distintos expertos que lo reprodujeron, los legisladores en la cámara y los médico-legistas en las publicaciones académicas, ubican a la víctima en un rol pasivo. Esa naturalización estaba anclada en la figura de las mujeres como patrimonio masculino, sin voluntad ni autonomía que explique el uso de sustancias.

\section{La mujer mundana, mujer de vida aireada}

La mujer de "la mala vida" conjugaba una serie de características que la convertirían en un factor de peligro para la salud. Hábitos como el "alcoholismo" y la vida relajada, conductas negativas tanto en varones como en mujeres, tenían un valor central para explicar el origen y la difusión de un conjunto de patologías ${ }^{(53,72)}$.

El escenario de la "mala vida" es el lugar en el que se entrecruzaban el delito y la locura $^{(72)}$, donde las mujeres ligadas a la prostitución estaban relacionadas con el uso de alcaloides. Un artículo del diario Crítica del 3 de enero de 1920, afirmaba que "la vuelta de coca" incluía a las "mujeres mundanas": "la que no se dope carece del más seductor atractivo sensual que brinda el repugnante y alambicado refinamiento del siglo" (citado por Bard en Los peligros de la Toxicomanía de 1923$)^{(3)}$.

La prostitución fue materia de análisis tanto de juristas como de médicos ${ }^{(73,74)}$. En 1908, Eusebio Gómez publica La mala vida en Buenos Aires. Allí definía la prostitución como un estado "equivalente o un derivativo de la criminalidad", una locura moral de base biológica. En su definición, las prostitutas eran criminales natas caracterizadas por "la ausencia de sentimientos maternales [...] la pasión por los licores, que llega a lo insaciable, y la falta más completa de pudor, que es la mayor de sus degeneraciones"(74).

A pesar del sesgo biologicista, el penalista señalaba la importancia de la educación moral y de atender a los determinantes del medio en la generación del mal. Cabe destacar de su conceptualización una distinción: entre las prostitutas extranjeras y las "criollas". A estas últimas, Gómez les atribuía valores positivos como tener "rasgos de nobleza", "pasión del amor verdadero" y "carencia por el afán de acumular dinero"; las caracteriza como más inestables emotivamente, presas fáciles de la sugestión y con afición por el alcohol ${ }^{(74)}$.

Esta diferenciación puede verse en dos historias citadas por Bard: por un lado, casos de mujeres en los que la prostitución era propia de la naturaleza degenerada, entre las que era claro su afán por contagiar a los débiles de espíritu tanto el vicio de la toxicomanía como la sífilis; mientras que, por otro, aparecen casos que se articularon con la noción de "degeneración adquirida", en los que una infracción de la ley paterna corrompe el espíritu y da lugar al uso de los tóxicos. Ambos permitirían neutralizar los argumentos de otros legisladores y médicos, que minimizaban el peligro de difusión de la toxicomanía.

\section{El caso de Juana Rosa}

Frustrado el primer intento en 1920 de prohibir el consumo de alcaloides sin prescripción médica, en 1923, Leopoldo Bard retomó el proyecto. En un artículo publicado en la revista Caras y Caretas, denunciaba el grave error de esa dilación causada por el clasismo de sus colegas, los cuales ubicaban a la toxicomanía como un cuadro que solo "se presentaba en cierta clase de mujeres". Para el nuevo periodo legislativo señalaba que la difusión se había convertido en un problema, en el que el único recurso era "una legislación represiva enérgica"(75). 
En la I Conferencia Sanitaria Nacional realizada en 1923, se llevó a cabo una sesión cuyo Orden del Día aglutinaba aspectos relativos a "Higiene Social. Profilaxis de la Sífilis. Reglamentación de la Prostitución. Vicios degenerativos tóxicos: alcohol, alcaloides y derivados del opio, la coca, etc." ${ }^{\prime(76)}$. Allí Bard logra el apoyo de destacados médicos como el propio Cabred y Gregorio Aráoz Alfaro, así como de referentes de distintas provincias.

Reglamentada en el último cuarto del siglo XIX, la prostitución se ejercía bajo supervisión sanitaria, como parte de las medidas de higiene social vinculadas a la sífilis. La amenaza de las enfermedades venéreas no se limitaba a elevar la mortalidad, sino también con propagarse al digno seno familiar. Los debates sobre la prostitución exponían los mecanismos por los cuales distintas fuerzas en pugna legitimaron las características socialmente aceptadas para la mujer: "digna de ver en público" y madre de los hijos de la nación ${ }^{(77)}$. Al igual que los peligros de la sífilis, el contacto con "mujeres de vida aireada" y las debilidades tóxicas de los "niños bien" amenazaban con intoxicar el seno familiar. En el periódico santafecino Nueva Época, del 25 de noviembre de 1922, se reportaba el caso dos varones de la clase distinguida "caídos bajo lo acción funesta del uso de la morfina [...] arrastrando la tradición de su apellido al banquillo de los reos"(3). El periodista, citado por Bard, reflexionaba:

...iqué puede esperase de damas y niñas que se encierran para morfinizarse? Basta formular la pregunta para estremecerse. Están en peligro las más tradicionales virtudes de la mujer argentina; y con ellas las de nuestro pueblo todo entero, que no es posible desconocer que la sociedad es lo que son las madres. Y madres que se están preparando en una soltería morfinómana, serán las engendradoras de la crápula hereditaria. El progreso que está adquiriendo el uso de la maldita droga en la juventud masculina $y$ en la mujer obligan a extremar todos los recursos para impedir que ella pueda estar, cual lo está hoy, al alcance de la mano. (Nueva Época, 25 de noviembre de 1922, citado por Bard)(3)

En la recopilación periodística realizada por Bard, en tanto evidencia que daba sustento a su proyecto prohibicionista, se describen varones "inconscientes" que frecuentaban cabarets, sitios de reunión de "mujeres de vida alegre y entre quienes el vicio de los alcaloides se halla más generalizado". Eran vástagos de las "clases acomodadas", inducidos al "vicio por aquellas mismas mujeres, cayendo así en la más humillante depravación moral y física" (La Argentina, 26 de agosto de 1922, citado por Bard) $)^{(3)}$.

El caso Juana Rosa, sucedido en Buenos Aires y publicado por El Diario del Plata de Uruguay el 27 de diciembre de 1922, se trató de una mujer de 23 años, soltera y que poseía libreta de permiso para ejercer la prostitución. Según la policía, había ingerido "una fuerte dosis de cocaína que le ocasionó la muerte". En los antecedentes policiales se constaba que "estaba sindicada como una de las infelices entregadas al abuso de los alcaloides, y en varias ocasiones tuvo entradas por sospechas de que se dedicaba al

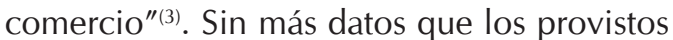
por el periódico, este caso le permitió a Bard articular prostitución y delito, junto con la idea de proselitismo toxicómano de las prostitutas, el cual explicaría cómo los varones débiles de espíritu caían presos de conductas degenerativas.

\section{La Mariposa: de digna virtud a maestra en el arte de suicidarse}

Con las figuras literarias como "La costurerita que dio el mal paso" y las milonguitas ${ }^{(78,79)}$, circulaban también en el imaginario colectivo los peligros de las conductas rebeldes. Esas figuras resaltaban las consecuencias sufridas por una joven virtuosa e inocente de barrio en la odisea emprendida en su "viaje al centro". Esa aventura, desembocaría irremediablemente en la vida nocturna y en la prostitución. Ese viaje puede asociarse a la figura de la prostituta criolla descripta por Gómez y que se repetirá en distintas expresiones 
culturales de la época ${ }^{(80)}$. A la prostituta crioIla se le reservaba una cuota de ingenuidad y docilidad que, si bien no explicaba, al menos justificaba el devenir vicioso.

Ejemplo de ese recorrido, el metafórico "viaje al centro", se refleja en el caso de La Mariposa. En 1923, el periódico La Montaña del 7 de junio de 1923 informaba que María Dolores Burgell, argentina de 25 años, había sido encontrada en una "casa de lenocinio" muerta a causa de intoxicación por cocaína. Según el relato del periodista, a los 18 años se había fugado de la casa paterna, contraviniendo la "oposición obstinada y violenta de sus padres" a que se case con un joven. Al año y medio de haberse casado, enviuda. El dolor de la pérdida la acercó a una amiga "ya iniciada en el vicio", quien la indujo "a buscar excitaciones y consuelos artificiales". A los 21 años, "rodaba, como muchas, en la pendiente fatal [...] su belleza de virgen y sus vicios de pecadora incorregible y satánica. [Era conocida] en el hampa por el apodo La Mariposa"(3).

A diferencia de los otros casos detallados, el de María Dolores articulaba una figura virtuosa que, frente a la contravención de la ley paterna, se ve condenada a un derrotero fatídico. En su proselitismo por la reforma prohibicionista, Bard busca evidenciar que el peligro de la toxicomanía no se limitaba ni a los sectores marginales ni a los hijos de la elite. El peligro era incluso para los florecientes sectores medios y proletarios urbanos. Sectores trabajadores, caracterizados por sus virtudes morales, se veían así expuestos a esta amenaza. La idea de "debilidad propia de la juventud" y, en especial, del caso de las mujeres, mostraba la urgencia de la reforma de corte represiva.

\section{CONCLUSIÓN}

Este trabajo ha buscado describir casos de mujeres que han utilizado sustancias psicoactivas entre fines del siglo XIX y las tres primeras décadas del siglo XX. Esos casos fueron expuestos por expertos de los ámbitos legislativo, médico-legal y periodístico de la Argentina. En un primer momento, en la época virreinal, los saberes expertos avanzaron, desprestigiando los conocimientos subalternos relacionados con la producción y administración de preparados. En esos discursos, la relación entre las mujeres y los usos de las drogas no se limitaba a su protagonismo como consumidoras, sino también en la producción y administración de fármacos en el ámbito doméstico y comunitario. El proceso de modernización del arte de curar tuvo como eje la problematización de esa relación entre mujeres y usos de fármacos. Supuso la cristalización de una clasificación, diferenciación y jerarquización de saberes, prácticas y sujetos.

Un segundo corrimiento en el proceso modernizador se da en el avance de las intervenciones médicas sobre el cuerpo femenino, en las que se multiplicaron los usos de fármacos. Fue la fase de una creciente medicalización de los eventos fisiológicos del cuerpo femenino y de un conjunto de dolencias para las cuales la prescripción de opiáceos fue central. Mitigar las dolencias asociadas a la "debilidad congénita" femenina no fue un proceso exclusivo de la Argentina, sino una tendencia común en los países occidentales ${ }^{(10,52)}$; tendencia que, a mediados del siglo XX, se podría vincular con la consolidación de la oferta de psicofármacos, dando lugar a una "tranquilidad recetada" protagonizada por mujeres ${ }^{(81,82)}$.

El cuerpo de la mujer era un objeto que se diseccionaba, se analizaba física y moralmente y sobre el que se daba un proceso de trabajo que posibilitaba el perfeccionamiento de técnicas y tecnologías. Allí las sustancias con efecto psicoactivo y los fármacos se convertían en auxiliares indispensables de la aventura médica de domesticar un cuerpo que se manifestaba como indomable. Su carne contenía un espíritu acechado por el peligro de la pasión desmedida, a la vez que era fuente de amor maternal. Fue la fase en la que se consolidaba la política de reducción de la mujer a una "matriz reproductora" de la raza ${ }^{(38,83)}$.

Cuando se tornó evidente "la apetencia por los alcaloides" en varones, pero también en algunas mujeres, el foco de atención de los especialistas y legisladores volvió a moverse. 
Se analizaron las prácticas asociadas a la automedicación, como el uso de los fármacos indicados en el tratamiento de la sífilis, pero también por su uso para inducir abortos. Los usos no autorizados de distintas drogas comenzaron a ser tema de debate. El eje común no fue el efecto psicoactivo, sino el uso sin supervisión médica y con fines cuestionados por los legisladores, por considerarlos inmorales y materia penable.

En las exposiciones ante la cámara, los legisladores presentaban a las mujeres como una figura difusa entre víctima y victimaria. Por un lado, las mujeres virtuosas, presas de un varón enfermo que facilitaba la disponibilidad de las sustancias. Por otro, la perversa mujer de la vida, una criminal nata, autora de "infanticidios", en cuya naturaleza estaba desvirtuar a los varones débiles de espíritu. Aun cuando las crónicas solo hablaban de sobredosis femeninas, era clara la preocupación por visibilizar los medios sociales en los que se daba la difusión de los tóxicos.
Para los autores anglosajones, la predisposición innata a la locura era propia de la mujer. Entre sus pares franceses, era poseedora de equilibrio inestable que no solo ponía en duda su racionalidad, sino que facilitaba su descrédito para reconocerla como ciudadana de pleno derecho ${ }^{(10)}$. En Argentina, las mujeres eran una amenaza para los especímenes masculinos débiles de espíritu, para el cuerpo social y para el progreso de la nación.

Para legitimar ese proceso se publicaron casos y se elaboraron explicaciones sobre la etiología de la toxicomanía. Recién con la tercera figura prototípica presentada por Bard, fue posible identificar un evento desencadenante, la contravención a la ley paterna, que dio lugar al proceso de "adquisición" del vicio degenerativo de la toxicomanía. Estos corrimientos permitieron que, en la sesión del 16 de julio de 1926, la Cámara de Diputados de la Nación Argentina sancionara la Ley 11331, dando inicio a casi un siglo de prohibicionismo $^{(84)}$.

\section{AGRADECIMIENTOS}

El presente trabajo es un resultado parcial de un proyecto de investigación más amplio desarrollado en el marco del Programa de Estudios Posdoctorales de la Universidad Nacional de Tres de Febrero. Dicho proyecto, titulado "Análisis de prácticas, saberes, sentidos y trayectorias de consumo de sustancias psicoactivas (SP) legales e ilegales de mujeres de 18 a 50 años pertenecientes a sectores medios del Área Metropolitana de Buenos Aires (AMBA)" fue financiado por la Agencia Nacional de Investigaciones Científicas y Tecnológicas del Ministerio de Ciencia y Técnica, a través del Préstamo BID-PICT 2977, otorgado en 2015.

\section{REFERENCIAS BIBLIOGRÁFICAS}

1. Cámara de Diputados Congreso de la Nación Argentina. Diario de Sesiones. 1920; Tomo II. Localizado en: Hemeroteca (Revistas), Biblioteca del Congreso de la Nación Argentina, Ciudad Autónoma de Buenos Aires.

2. Fernández H, Jones A, Brandam J, Pacheco D. La morfinomanía ante la ley penal: Informe pericial. Revista de Criminología, Psiquiatría y Medicina Legal. 1918;V:555-568. Localizado en: Catálogo General, Biblioteca Central "Juan José Montes de Oca", Facultad de Medicina, Universidad de Buenos Aires, Ciudad Autónoma de Buenos Aires. 
3. Bard L. Los peligros de la Toxicomanía: Proyecto de ley para la represión del abuso de los alcaloides. Buenos Aires: Talleres Gráficos Argentinos de L. J. Rosso y Cía.; 1923. Localizado en: Colección General, Biblioteca del Congreso de la Nación Argentina, Ciudad Autónoma de Buenos Aires.

4. Weissmann P. Degenerados y viciosos: Primeras conceptualizaciones acerca de las toxicomanías en la $\mathrm{Ar}$ gentina. Temas de Historia de la Psiquiatría Argentina. 2001;12:1-8.

5. Cámara de Senadores del Congreso de la Nación Argentina. Diario de Sesiones. 1920; Tomo IV. Localizado en: Hemeroteca (Revistas), Biblioteca del Congreso de la Nación Argentina, Ciudad Autónoma de Buenos Aires.

6. Weissmann P. Toxicomanias. Mar del Plata: Universidad Nacional de Mar del Plata; 2002.

7. Sánchez Antelo V. Primeros debates sobre legislación del uso de drogas en Argentina fines del Siglo XIX y comienzos del XX: la propuesta del Dr. Leopoldo Bard y su contexto socio-histórico. Salud Colectiva. 2012;8(3):275-286.

8. O'Malley P, Valverde M. Pleasure, freedom and drugs: The uses of 'pleasure' in liberal governance of drug and alcohol consumption. Sociology. 2004;38(1):25-42.

9. Seddon T. Women, harm reduction and history: gender perspectives on the emergence of the "British System" of drug control. International Journal on Drug Policy. 2008;19(2):99-105. doi: 10.1016/j.drugpo.2007.10.004.

10. Davenport-Hines R. La búsqueda del olvido: Historia global de las drogas, 1500-2000. Barcelona: Fondo de Cultura Económica; 2003.

11. Giacomello C. Mujeres, delitos de drogas y sistemas penitenciarios en América Latina. Revista Pensamiento Penal [Internet]. 2013 [citado 10 mar 2019]. Disponible en: https://tinyurl.com/y8ln9q9t.

12. Almeda Samaranch E, Di Nella D. Mujeres y cárceles en América Latina: Perspectivas críticas y feministas. Papers: Revista de Sociología. 2017;102(2):183-214. doi: 10.5565/rev/papers.2335.

13. Youngers $\mathrm{C}$. ¿Cómo incorporar una perspectiva de género en las políticas de drogas?: la experiencia de Uruguay [Internet]. 2016 [citado 10 mar 2019]. Disponible en: https://tinyurl.com/y7egttcz.

14. Measham F. "Doin gender"-“Doing drugs": conceptualizing the gendering of drugs cultures. Contemporary Drug Problems. 2002;29(2):335-373.

15. Campbell ND, Ettorre E. Gendering addiction: The politics of drug treatment in a neurochemical world. London: Palgrave Macmillan; 2011.

16. Ettorre E. Embodied deviance, gender, and epistemologies of ignorance: Re-visioning drugs use in a neurochemical, unjust world. Substance Use \& Misuse. 2015;50(6): 794-805. doi: 10.3109/10826084.2015.978649.
17. Ettorre E. Revisioning women and drug use: Gender, power and the body. London: Palgrave Macmillan; 2007.

18. Romo N, Camarotti AC, Tarragona A, Touris C. Doing gender in a toxic world: Women and freebase cocaine in the City of Buenos Aires (Argentina). Substance Use \& Misuse. 2015;50(5):557-565. doi: 10.3109/ 10826084.2014 .991404$.

19. Pecheny M, Hiller R, Manzelli H, Binstock G. Mujeres, infección por $\mathrm{VIH}$ y uso de drogas en la Argentina reciente. En: Epele $\mathrm{M}$, (comp.). Padecer, cuidar y tratar: Estudios socio-antropológicos sobre consumo problemático de drogas. Buenos Aires: Antropofagia; 2012. p. 25-55

20. Sánchez Antelo V. Drogas: entre cuerpos regulados y morales desviadas. Argentina, 1880-1960. Anuario Colombiano de Historia Social y de la Cultura. 2018;45(1):315338. doi: 10.15446/achsc.v45n1.67561.

21. Aureano GR. La construction politique du toxicomane dans I'Argentine post-autoritaire: Un cas de citoyenneté à basse intensité [Thèse doctoral]. Montreal: Univesité de Montréal; 1998.

22. Vigarello G. Lo sano y lo malsano, desde la edad media hasta nuestros días. Madrid: Abada; 2006.

23. Romaní O, Comelles JM. Les contradictions liées à I'usage des psychotrops dans les sociétés contemporaines: automédication et dépendance. Psychotropes. $1991 ; \mathrm{VI}(3): 39-57$

24. Armus D. Medicina casera, remedios y curanderos en los inicios de la medicalización de la ciudad moderna: Buenos Aires, 1870-1940. Tempos Históricos. 2016;20(1):47-80.

25. Beltrán JR. Historia del Protomedicato de Buenos Aires. Buenos Aires: El Ateneo; 1937. Localizado en: Colección General, Biblioteca del Congreso de la Nación Argentina, Ciudad Autónoma de Buenos Aires.

26. Di Liscia MS. Itinerarios curativos: Saberes, terapias y prácticas médicas indígenas, populares y científicas (Región pampeana, 1750-1910). [Tesis doctoral]. Madrid: Universidad Complutense de Madrid; 2000.

27. Torres SG. Normas complementarias: Estupefacientes, Ley 23.737. En: Baigún D, Zaffaroni ER, (dirs), De Langhe M, Terragni MA, (coords). Código Penal y normas complementarias: Análisis y jurisprudencia. Buenos Aires: Hammurabi; 2014.

28. Cámara de Senadores. Congreso de la Nación Argentina. Diario de Sesiones. 1897. Localizado en: Hemeroteca (Revistas), Biblioteca del Congreso de la Nación Argentina, Ciudad Autónoma de Buenos Aires.

29. Cámara de Diputados Congreso de la Nación Argentina. Diario de Sesiones. 1902. Localizado en: Hemeroteca (Revistas), Biblioteca del Congreso de la Nación Argentina, Ciudad Autónoma de Buenos Aires.

30. Martín AL. Parir, cuidar y asistir: El trabajo de las parteras y enfermeras en Buenos Aires (1877-1955). [Tesis 
doctoral]. Buenos Aires: Facultad de Filosofía y Letras, Universidad de Buenos Aires; 2014.

31. Ingenieros J. La locura en Argentina. Buenos Aires: Cooperativa Editorial "Buenos Aires"; 1920. Localizado en: Colección General, Biblioteca del Congreso de la Nación Argentina, Ciudad Autónoma de Buenos Aires.

32. Knibiehler Y. Historia de las madres y de la maternidad en Occidente. Buenos Aires: Nueva Visión; 2001.

33. Goitía F. Contribución al estudio de la higiene del embarazo. [Tesis doctoral]. Buenos Aires: La Semana Médica, Imprenta de Obras de E. Spinelli; 1908. Localizado en: Catálogo General, Biblioteca Central "Juan José Montes de Oca", Facultad de Medicina, Universidad de Buenos Aires, Ciudad Autónoma de Buenos Aires.

34. Alurralde M. La Galvanización: Tratamiento de los vómitos incoercibles. Anales del Círculo Médico Argentino. 1895;XVII(19):493-500. Localizado en: Catálogo General, Biblioteca Central "Juan José Montes de Oca", Facultad de Medicina, Universidad de Buenos Aires, Ciudad Autónoma de Buenos Aires.

35. Moguillansky J. Cesárea vaginal. Capital Federal: Universidad Nacional de Buenos Aires; 1918. Localizado en: Catálogo General, Biblioteca Central "Juan José Montes de Oca", Facultad de Medicina, Universidad de Buenos Aires, Ciudad Autónoma de Buenos Aires.

36. Villarroel L. Examen médico en obstetricia. [Tesis doctoral]. Buenos Aires: Facultad de Ciencias Médicas, Universidad Nacional de Buenos Aires, Editora Etchepareborda; 1903. Localizado en: Catálogo General, Biblioteca Central "Juan José Montes de Oca", Facultad de Medicina, Universidad de Buenos Aires, Ciudad Autónoma de Buenos Aires.

37. La ciencia argentina. Un gran descubrimiento. Caras y Caretas. 1915;865:1-5. Localizado en: Hemeroteca Digital, Biblioteca Nacional de España.

38. Knibiehler Y, Fouquet $C$. La femme et les médecins: Analyse historique. Paris: Hachette; 1983.

39. Vallejo G, Miranda M. Los saberes del poder: Eugenesia y biotipología en la Argentina del siglo XX. Revista de Indias. 2004;64(231):425-444. doi: 10.3989/ revindias.2004.i231.547.

40. Roth C. From free womb to criminalized woman: fertility control in Brazilian slavery and freedom. Slavery \& Abolition. 2017;38(2):269-286. doi: 10.1080/0144039X.2017.1316965.

41. Roth C. Policing Pregnancy: reproduction, poverty, and the law in early twentieth-century Rio de Janeiro. Journal of Women's History. 2017;29(4):85-108. doi: 10.1353/jowh.2017.0052.

42. Biernat C. Jueces, médicos y enfermos: Prácticas y sentidos en la construcción social del delito de contagio venéreo en la Argentina durante la primera mitad del siglo XX. História (São Paulo). 2019;38:1-32. doi: 10.1590/1980-4369e2019050.
43. Armus D. Eugenics in Buenos Aires: Discourses, practices, and historiography. História, Ciências, Saúde-Manguinhos. 2016;23(Suppl 1):S149-S170. doi: 10.1590/ s0104-59702016000500009.

44. Ben P. Cuerpos femeninos y cuerpos abyectos: La construcción anatómica de la feminidad en la medicina argentina. En: Gil Lozano F, Pita VP, Ini MG, (eds.). Historia de las mujeres en la Argentina. Tomo I, Colonia y siglo XIX. Buenos Aires: Taurus; 2000. p. 253-267.

45. Ledesma Prietto N. Entre la mujer y la madre: Discursos médicos y la construcción de normas de género (Argentina, 1930-1940). Trabajos y Comunicaciones. 2015;(42):1-15.

46. Castells FC. La temibilidad femenina en los discursos médico-legales argentinos (1902-1913). Revista de Historia del Derecho. 2017;(54):25-51.

47. Peralta A. Ensayo sobre la Clorosis. [Tesis doctoral]. Buenos Aires: Imprenta de Arzac; 1847. Localizado en: Catálogo General, Biblioteca Central "Juan José Montes de Oca", Facultad de Medicina, Universidad de Buenos Aires, Ciudad Autónoma de Buenos Aires.

48. Fournol, Heiser, Samne. Mi médico: Guía práctica de Medicina e higiene. Tomo I. Buenos Aires: Editorial Labor; 1930.

49. Figueroa M. De las causas del aborto. [Tesis doctoral]. Buenos Aires: Facultad de Ciencias Médicas, Universidad Nacional de Buenos Aires, El Demócrata; 1882. Localizado en: Catálogo General, Biblioteca Central "Juan José Montes de Oca", Facultad de Medicina, Universidad de Buenos Aires, Ciudad Autónoma de Buenos Aires.

50. Bozetti A. Inyecciones vaginales. [Tesis doctoral]. Buenos Aires: Facultad de Ciencias Médicas, Universidad de la Capital, Imprenta Biedma; 1887. Localizado en: Catálogo General, Biblioteca Central "Juan José Montes de Oca", Facultad de Medicina, Universidad de Buenos Aires, Ciudad Autónoma de Buenos Aires.

51. Ariès $P$, Duby G. Historia de la vida privada: De la Revolución Francesa a la Primera Guerra Mundial. 3a ed. Barcelona: Taurus; 2017.

52. Knibiehler Y. Cuerpos y corazones. En: Historia de las mujeres: El siglo XIX. Madrid: Turus; 2000. p. 339-388.

53. Salessi J. Médicos maleantes y maricas: Higiene, criminología y homosexualidad en la construcción de la nación argentina, (Buenos Aires: 1871-1914). Buenos Aires: Beatriz Viterbo; 1997.

54. Calderón J. Moralidad comparada del hombre y de la muger bajo el punto de vista penal. [Tesis doctoral]. Buenos Aires: Imprenta de M. Biedma; 1878. Localizado en: Colección Tesis, Biblioteca Central, Facultad de Derecho, Universidad de Buenos Aires, Ciudad Autónoma de Buenos Aires.

55. Biernat C. La eugenesia argentina y el debate sobre el crecimiento de la población en los años de entreguerras. Cuadernos del Sur, Historia. 2005;(34):251-273. 
56. Cabred D. Contribución al estudio de la locura refleja. [Tesis doctoral]. Buenos Aires: Facultad de Ciencias Médicas, Universidad Nacional de Buenos Aires, Imprenta de obras La Nación; 1881. Localizado en: Catálogo General, Biblioteca Central "Juan José Montes de Oca", Facultad de Medicina, Universidad de Buenos Aires, Ciudad Autónoma de Buenos Aires.

57. Weissmann P. Morfinomanía y defensa social. Revista de la Asociación Española de Neuropsiquiatría. 2001;XXI(78):113-123.

58. Vallejo MS. Cuerpos histéricos en la medicina de Buenos Aires (1877-1900): Teorías, representaciones y dispositivos clínicos. Historia (Santiago). 2019;52(1):139167. doi: 10.4067/S0717-71942019000100139.

59. Didi-Huberman G. La invención de la histeria Charcot y la iconografía fotográfica de la Salpetriere. Madrid: Cátedra; 2007.

60. Firmart IJ. Notas sobre el tratamiento de la Histeria: Tesis inaugural. Buenos Aires: Facultad de Ciencias Médicas, Universidad de la Capital, Imprenta, Litografía y Encuadernación de Jacobo Peuser; 1889. Localizado en: Catálogo General, Biblioteca Central "Juan José Montes de Oca", Facultad de Medicina, Universidad de Buenos Aires, Ciudad Autónoma de Buenos Aires.

61. Arce C. Curabilidad de la locura en el Manicomio de Mujeres. [Tesis doctoral]. Buenos Aires: Imprenta y Litografía La Argentina; 1881. Localizado en: Catálogo General, Biblioteca Central "Juan José Montes de Oca", Facultad de Medicina, Universidad de Buenos Aires, Ciudad Autónoma de Buenos Aires.

62. Ferrand A. La histeria. [Tesis doctoral]. Buenos Aires: Imprenta Moreno; 1888. Localizado en: Catálogo General, Biblioteca Central "Juan José Montes de Oca", Facultad de Medicina, Universidad de Buenos Aires, Ciudad Autónoma de Buenos Aires.

63. Yzaurralde J. Histeria. [Tesis doctoral]. Buenos Aires: Imprenta y encuadernación San Martín; 1889. Localizado en: Catálogo General, Biblioteca Central "Juan José Montes de Oca", Facultad de Medicina, Universidad de Buenos Aires, Ciudad Autónoma de Buenos Aires.

64. Doyle E. Histeria e intoxicación mercurial. [Tesis doctoral]. Buenos Aires: Facultad de Ciencias Médicas: Imprenta y Librería Boullosa; 1900. Localizado en: Catálogo General, Biblioteca Central "Juan José Montes de Oca", Facultad de Medicina, Universidad de Buenos Aires, Ciudad Autónoma de Buenos Aires.

65. Almeida A. La morfinomanía. [Tesis doctoral]. Buenos Aires: Facultad de Ciencias Médicas, Universidad Nacional de Buenos Aires, Establecimiento Tipográfico El Censor; 1891. Localizado en: Catálogo General, Biblioteca Central "Juan José Montes de Oca", Facultad de Medicina, Universidad de Buenos Aires, Ciudad Autónoma de Buenos Aires.

66. Bard L. Respuesta a la encuesta sobre el problema de la toxicomanía. Revista de la Asociación Médica Argentina. 1923:740-745. Localizado en: Catálogo Ge- neral, Biblioteca Central "Juan José Montes de Oca", Facultad de Medicina, Universidad de Buenos Aires, Ciudad Autónoma de Buenos Aires.

67. Bard L. Una encuesta internacional a propósito de la lucha contra la toxicomanía. Revista de la Asociación Médica Argentina. 1923;620-626; 1183-1189; 13221332. Localizado en: Catálogo General, Biblioteca Central "Juan José Montes de Oca", Facultad de Medicina, Universidad de Buenos Aires, Ciudad Autónoma de Buenos Aires.

68. Barrancos D. Mujeres en la sociedad argentina: Una historia de cinco siglos. 2a ed. Buenos Aires: Sudamericana; 2010.

69. Guy DJ. Public health, gender, and private morality: Paid labor and the formation of the body politic in Buenos Aires. Gender \& History. 1990;2(3):297-318. doi. 10.1111/j.1468-0424.1990.tb00102.x.

70. Bermann G. Toxicomanías. Córdoba: El Ateneo; 1926.

71. Nari M. Maternidad, política y feminismo. En: Historia de las mujeres en la Argentina. Tomo II: Siglo XX. Buenos Aires: Aguilar; 2000. p. 197-221.

72. Dovio M. Representaciones sobre mujeres de "mala vida" en la revista Archivos de Psiquiatría, Criminología, Medicina Legal y Ciencias Afines y Revista de Criminología, Psiquiatría y Medicina Legal (1902-1935). En: Mujeres en cuestión: Escrituras, ideologías y cuerpos. 2012. p. $97-140$

73. Ingenieros J. Criminología. Madrid; Daniel Jorro Editor; 1913. Localizado en: Colección General, Biblioteca del Congreso de la Nación Argentina, Ciudad Autónoma de Buenos Aires.

74. Gómez E. La mala vida en Buenos Aires (1908). Buenos Aires: Biblioteca Nacional; 2011.

75. Bard L. La difusión de la toxicomanía: La apetencia por los alcaloides. Caras y Caretas. 1923;(1292):7. Localizado en: Catálogo General, Biblioteca Central "Juan José Montes de Oca", Facultad de Medicina, Universidad de Buenos Aires, Ciudad Autónoma de Buenos Aires.

76. Departamento Nacional de Higiene. Conferencia Sanitaria Nacional: Antecedentes, sesiones y conclusiones. Buenos Aires: Talleres Gráficos Editorial Argentina de Ciencias Políticas; 1923. Localizado en: Catálogo general, Biblioteca Central "Juan José Montes de Oca", Facultad de Medicina, Universidad de Buenos Aires, Ciudad Autónoma de Buenos Aires.

77. Guy DJ. El sexo peligroso: La prostitución Legal en Buenos Aires 1875-1955. Buenos Aires: Sudamericana; 1994.

78. Armus D. La ciudad impura: salud, tuberculosis y cultura en Buenos Aires, 1870-1950. Buenos Aires: Edhasa; 2007.

79. Armus D. El viaje al centro: “Tísicas, costureritas y milonguitas en Buenos Aires, 1910-1940." Salud Colectiva. 2005; 1(1):79-96. 
80. Tossounian C. Milonguitas: Tango, gender and consumption in Buenos Aires (1920-1940). Estudios Interdisciplinarios de América Latina y El Caribe. 2016;27(2):29-45.

81. Burin M. La mujer y los estados depresivos. En: El malestar de las mujeres, la tranquilidad recetada. México DF: Paidós; 2000. p. 112-119.

82. Romo N. La mirada de género en el abordaje de los usos y abusos de drogas. Revista Española de Drogodependencia. 2010;35(3):269-272.
83. Ettorre E. Revisioning women and drug use: gender sensitivity, embodiment and reducing harm. International Journal of Drug Policy.2004;15(5):327-335.

84. Cámara de Diputados del Congreso de la Nación. Diario de Sesiones. Buenos Aires; 1926. Localizado en: Hemeroteca (Revistas), Biblioteca del Congreso de la Nación Argentina, Ciudad Autónoma de Buenos Aires.

\section{FORMA DE CITAR}

Sánchez Antelo V. Mujeres argentinas y usos de drogas: análisis sociohistórico del uso femenino de sustancias psicoactivas en Argentina (1860-1930). Salud Colectiva. 2020;16:e2446 doi: 10.18294/sc.2020.2446.

Recibido: 6 ago 2019 | Versión final: 31 mar 2020 | Aprobado: 14 abr 2020 | Publicado en línea: 4 may 2020

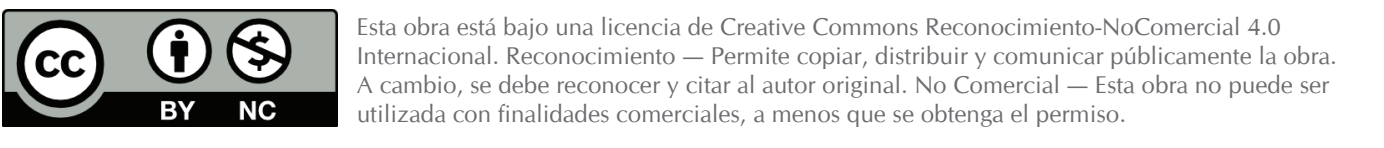

https://doi.org/10.18294/sc.2020.2446 